\title{
High Sensitive Refractive Index Sensor Based on Cladding Etched Photonic Crystal Fiber Mach-Zehnder Interferometer
}

\author{
Haifeng DU, Xiaoyan SUN, Youwang HU*, Xinran DONG, and Jianhang ZHOU \\ State Key Laboratory of High Performance Complex Manufacturing, College of Mechanical and Electrical Engineering, \\ Central South University, Changsha 410083, China \\ *Corresponding author: Youwang HU_ E-mail: huyw@csu.edu.cn
}

\begin{abstract}
A high sensitive refractive index sensor based on the cladding etched photonic crystal fiber (PCF) Mach-Zehnder interferometer (MZI) is proposed, which is spliced a section of photonic crystal fiber between two single modes fibers (SMFs). The interference fringe of the MZI shifts with the variation of the ambient refractive index (RI). It is found that the RI sensitivity slightly decrease with an increase in the interference length. The sensitivities of MZI with $35 \mathrm{~mm}$ PCF, $40 \mathrm{~mm}$ PCF, and $45 \mathrm{~mm}$ PCF are $106.19 \mathrm{~nm} / \mathrm{RIU}, 93.33 \mathrm{~nm} / \mathrm{RIU}$, and $73.64 \mathrm{~nm} / \mathrm{RIU}$, respectively, in the range of 1.333 to 1.381 . After etched, the RI sensitivity of the MZI could be improved obviously. The RI sensitivities of the MZI with $35 \mathrm{~mm}$ PCF are up to $211.53 \mathrm{~nm} / \mathrm{RIU}$ and $359.37 \mathrm{~nm} / \mathrm{RIU}$ when the cladding diameter decreases to $112 \mu \mathrm{m}$ and $91 \mu \mathrm{m}$, respectively. Moreover, the sensor is insensitive to temperature, and the measured sensitivity is only $9.21 \mathrm{pm} /{ }^{\circ} \mathrm{C}$ with the range from $20{ }^{\circ} \mathrm{C}$ to $500{ }^{\circ} \mathrm{C}$. In addition, the sensor has advantage of simple fabrication, low cost, and high RI sensitivity.
\end{abstract}

Keywords: Refractive index sensor; Mach-Zehnder interferometer; photonic crystal fiber; hydrofluoric acid

Citation: Haifeng DU, Xiaoyan SUN, Youwang HU, Xinran DONG, and Jianhang ZHOU, "High Sensitive Refractive Index Sensor Based on Cladding Etched Photonic Crystal Fiber Mach-Zehnder Interferometer," Photonic Sensors, 2019, 9(2): $126-134$.

\section{Introduction}

Refractive index sensors can be used to measure the concentrations of gases and liquids, and are widely used in the biological and chemical environmental detection $[1,2]$. There are two optical methods, the optical path measurement method and optical fiber measurement method, which are used to measure the refractive indices of liquids [3]. The optical path structure is complex, and the optical path is not stable. Optical fiber is widely used in the liquid refractive index test because of its light weight, small volume, strong electromagnetic interference resistance, and simple design [4]. Up to now, a number of optical fiber microstructures have been proposed for refractive index sensors, including long period fiber grating (LPFG) $[5,6]$, fiber Bragg grating (FBG) [7], Fabry-Perot interferometer (FPI) [8], and Mach-Zehnder interferometer (MZI) [9, 10]. These structures have high sensitivity in refractive index sensing. However, a serious disadvantage for the above structures is high sensitivity to the environmental temperature $[11,12]$. Unlike traditional polarization maintaining photonic crystal fibers (PM-PCF), which contain at least two different glasses each with a different thermal expansion coefficient, the photonic crystal fiber birefringence is highly insensitive to the

Received: 27 July 2018 / Revised: 29 November 2018

(C) The Author(s) 2019. This article is published with open access at Springerlink.com DOI: 10.1007/s13320-019-0532-2

Article type: Regular 
temperature $[4,13]$. The optical fiber sensor based on the MZI with the PCF structure has the advantages of ease to fabricate and convenience to measure, which is a common method in the research of refractive index sensors.

In recent years, many novel in-line MZIs with the PCF structure have been proposed and studied. Y. Zhao et al. [14] proposed a PCF interferometer structure with up-tapered joints for RI sensing, which achieved a high RI sensitivity of $252 \mathrm{~nm} / \mathrm{RIU}$ in the RI range of $1.333-1.379$. D. Wu et al. [15] proposed an RI sensor based on a tapered PCF MZI structure with an RI sensitivity of $51.902 \mathrm{~nm} / \mathrm{RIU}$ in the range 1.3411 - 1.3737. Y. Zhao et al. [16] reported a novel refractive index (RI) sensor based on the PCF-MZI which is fabricated by cascading a section of PCF with half-taper collapse regions (HTCRs) between two single mode fibers (SMFs) and investigated the RI sensitivity of $181.96 \mathrm{~nm} / \mathrm{RIU}$ when the RI varied from 1.3333 to 1.3574 . Dnyandeo Pawar et al. [17] proposed a tapered polarization maintaining photonic crystal fiber (PM-PCF) MZI sensor, and it had a sensitivity of $20.53 \mathrm{~nm} / \mathrm{RIU}$ within the RI range from 1.33 to 1.37 . These special structures of the MZI including tapered and cascading structure are complex to fabricate, time-consuming, and with low sensitivity. In order to improve the sensitivity of optical fiber sensors, many novel methods are proposed, such as chemical etching and coating on the optical fiber surface. Y. C. Tan et al. [18] presented a carbon nanotubes (CNT) deposited PCF featuring a Mach-Zehnder interferometer configuration for refractive index (RI) sensing. L. Melo et al. [19] reported an in-line MZI coated with hafnium oxide by atomic layer deposition to increase the RI sensitivity which was $1307 \mathrm{~nm} / \mathrm{SRI}$ within the range of 1.3327 - 1.3634. However, the deposition techniques used usually involve highly precise equipment or a long fabrication duration due to the extensive amounts of chemical processes involved.
In this paper, we present a PCF MZI refractive index sensor, which is realized by splicing a piece of PCF between two standard single mode fibers with a fusion splicer. The result shows that the effective RI of high order modes of the PCF cladding is more sensitive and more linear to surrounding refractive index changing, so we select high order mode spectra as the experimental measurement data. With an increase in the PCF length $(30 \mathrm{~mm}, 35 \mathrm{~mm}$, and $40 \mathrm{~mm}$ ), the RI sensitivity decreases gradually $(106.19 \mathrm{~nm} / \mathrm{RIU}, 93.33 \mathrm{~nm} / \mathrm{RIU}$, and $73.64 \mathrm{~nm} / \mathrm{RIU})$ within the RI range of 1.333 - 1.381. Afterwards, we adopt a novel method of etching the surface of photonic crystal fiber (the PCF length of $35 \mathrm{~mm}$ ) by using hydrofluoric acid (HF) to enhance the RI sensitivity. The refractive index sensitivity increases to $359.37 \mathrm{~nm} / \mathrm{RIU}$ when the cladding diameter decreases to $91 \mu \mathrm{m}$. With a decrease in the cladding diameter, the sensitivity of refractive index will be improved obviously. In addition, the PCF-MZI has advantages of temperature insensitivity, simple fabrication, low cost, and high RI sensitivity.

\section{Sensor fabrication and principle}

\subsection{Design of MZI}

The schematic of the sensor structure is shown in Fig. 1. The sensor is composed of SMFs and one segment of PCF (NKT Photonics LMA-10). The PCF is sandwiched to SMFs (Corning SMF-28). They are spliced together directly by a commercial fusion splicer (Fujikura $80 \mathrm{~S}$ ), and the splicing loss is about $1 \mathrm{~dB}$. Figure 1(b) shows the scanning electron micrograph (SEM) of the cross section of the PCF. The PCF has an outside diameter of $125 \mu \mathrm{m}$, a core diameter of $10 \mu \mathrm{m}$, a hole diameter of $2.85 \mu \mathrm{m}$, and a hole pitch of $7 \mu \mathrm{m}$. The standard single mode fiber has an outside diameter of $125 \mu \mathrm{m}$ and a core diameter of $9 \mu \mathrm{m}$. Figures 1(a) and 1(c) show the schematic diagram and optical micrograph of the MZI with the PCF structure. Through many experimental results, we find that a high fringe 
contrast suitable for fusion parameters can be obtained by tuning the arc power and arc time are +60 bit (the discharge intensity of standard is 0 ) and $380 \mathrm{~ms}$, and the overlap value is $20 \mu \mathrm{m}$, respectively. From the collapsed region in Fig. 1(c), we can know that the air holes of the PCF have slight collapse. However, the collapse of air holes will increase the mode field diameter and make the energy between the two modes of interference more balanced, which is more conducive to the formation of interference and increase the contrast of spectral fringes [20,21]. A light source and an optical spectrum analyzer are connected to monitor the spectrum change during the fabrication.
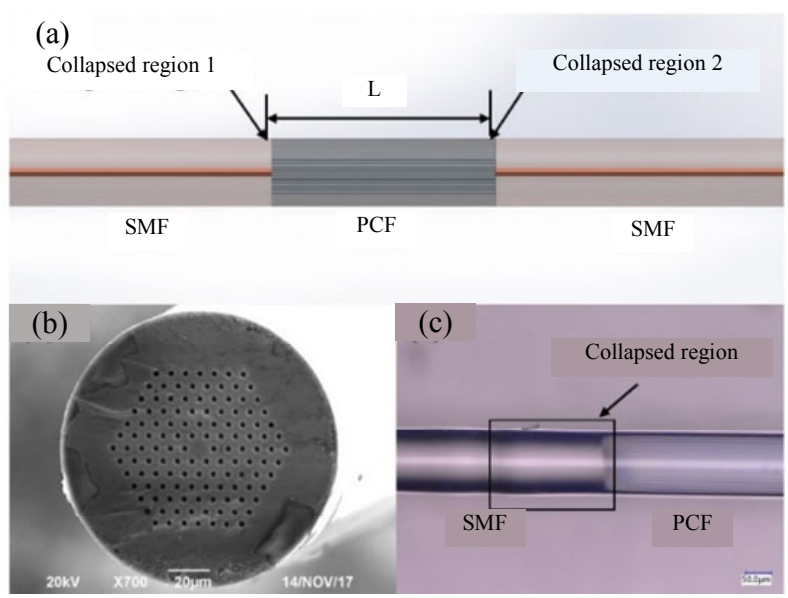

Fig. 1 In-line MZI with the PCF structure (a) schematic diagram of the MZI, (b) scanning electron micrograph (SEM) of the cross section of a LMA-10-PCF, and (c) optical micrograph of the MZI.

\subsection{Principle of the MZI}

Figure 1 shows the in-line MZI with the PCF structure. A piece of PCF is spliced between two standard single mode fibers directly. When light travels from the SMF to the PCF, the fundamental mode of the SMF begins to diffract. In the first collapsed region, both the core and cladding modes with different propagation constants are excited. The interference spectrum can be expressed using the following two-beam optical interference equation $[14,22]$ :

$$
I=I_{1}+I_{2}+\sqrt{I_{1} I_{2}} \cos \beta
$$

where $I$ is the intensity of the total interference signal, and $I_{1}$ and $I_{2}$ are the intensities of the core and cladding modes, respectively. The phase difference $\beta$ between the core and clad modes of an MZI is expressed as

$$
\beta=\frac{2 \pi \Delta n_{\mathrm{eff}} L}{\lambda}
$$

where $L$ is the interference length, $\lambda$ is the wavelength of propagating light, and $\Delta n_{\text {eff }}$ is the difference between the effective refractive indices of core and cladding modes, $\Delta n_{\text {eff }}=n_{\text {eff }}^{\text {core }}-n_{\text {eff }}^{\text {clad }}$. When the difference between the cladding mode and core mode equals to $(2 m+1) \pi$, the $m$-order interference valley is shown as follows:

$$
\lambda_{m}=\frac{2 \Delta n_{\mathrm{eff}} L}{2 m+1} .
$$

The free spectrum range (FSR) of the interference spectrum can be defined as follows:

$$
\Lambda=\frac{\lambda^{2}}{\Delta n_{\text {eff }} L} .
$$

The wavelength of the $m$-order interference valley varies along with the surrounding refractive index, and the variation is shown as

$$
\Delta \lambda_{m}=\frac{2\left(\Delta n_{\text {eff }}+\Delta n\right) L}{2 m+1}-\frac{2 \Delta n_{\text {eff }} L}{2 m+1}=\frac{2 \Delta n L}{2 m+1}
$$

where $\Delta n$ is the variation of the effective refractive index of the photonic crystal fiber cladding along with surrounding refractive index changing.

\subsection{Experimental measurement method}

The schematic diagram of our experimental measurement is shown in Fig. 2. A broadband light source $(\mathrm{C}+\mathrm{L}$, Shanghai Hanyu) with a wavelength range of $1528 \mathrm{~nm}-1602 \mathrm{~nm}$ is launched into the MZI structure. There is an optical path difference when light transmits through the sensor, and the interference will happen at the second collapsed region. The interference spectrum can be monitored by using an optical spectrum analyzer (OSA, Agilent $86142 \mathrm{~B}, 600 \mathrm{~nm}-1700 \mathrm{~nm})$. The sensor is attached to the bottom of the glassware during testing the RI changes. 


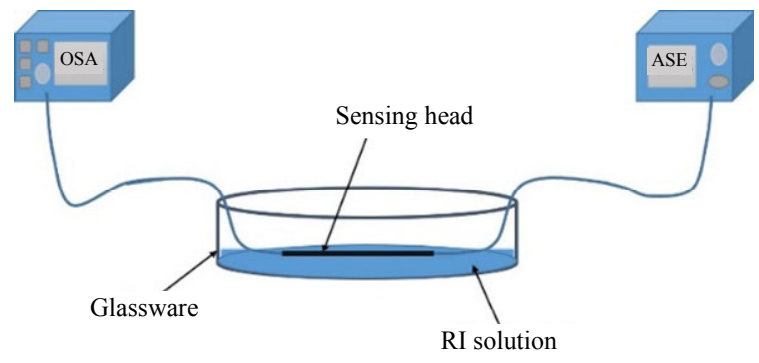

Fig. 2 Schematic diagram of the experimental measurement.

\section{Results and discussion}

Different parameters influence the properties and performances of the PCF interferometer, such as arc time, arc power, interference length, and the diameter of the PCF. These factors should be considered during the fabrication of the PCF interferometer. In this paper, the effects of the interference length on the interference spectrum and the sensitivity of RI sensing are investigated experimentally. Three kinds of PCF interferometer with different interference lengths of $30 \mathrm{~mm}, 35 \mathrm{~mm}$, and $40 \mathrm{~mm}$ are made by using the same welding parameters. The transmission spectra of a PCF interferometer with different sensing lengths in air and water are shown in Fig.3. From Fig. 3, we can see some interference peaks with the low fringe contrast when the sensor is immersed in air, which are caused by multimode interference. When the sensor is immersed in water, the wavelength is red-shift, and the interference peaks caused by multimode interference are decreased because the refractive index of water is higher than that of air, and the multimode interference is suppressed in water. The interference peaks produced by multi-mode interference have a small influence on sensing.

In air, $\lambda_{m}$ (the wavelength of the $m$-order interference valley) is $1585.74 \mathrm{~nm}, 1575.68 \mathrm{~nm}$, and $1583.81 \mathrm{~nm}$, and the fringe spaces ranges $(\Lambda)$ for the three PCF interferometers with the interference length of $30 \mathrm{~mm}, 35 \mathrm{~mm}$, and $40 \mathrm{~mm}$ are $25.5 \mathrm{~nm}$, $18.9 \mathrm{~nm}$, and $16.2 \mathrm{~nm}$, respectively. This result shows that the average fringe period decreases with an increase in the interferometer length, which is in conformity with the theory of interference in (4). According to (3) and (4), we can calculate the $\Delta n_{\text {eff }}$ $=0.0033,0.0038$, and 0.0039 , and $m=62,84$, and 98 for the PCF lengths of $30 \mathrm{~mm}, 35 \mathrm{~mm}$, and $40 \mathrm{~mm}$, respectively. According to (5), we know that the refractive index sensitivity is proportional to the PCF length $(L)$ and inversely proportional to interference mode $(m)$.

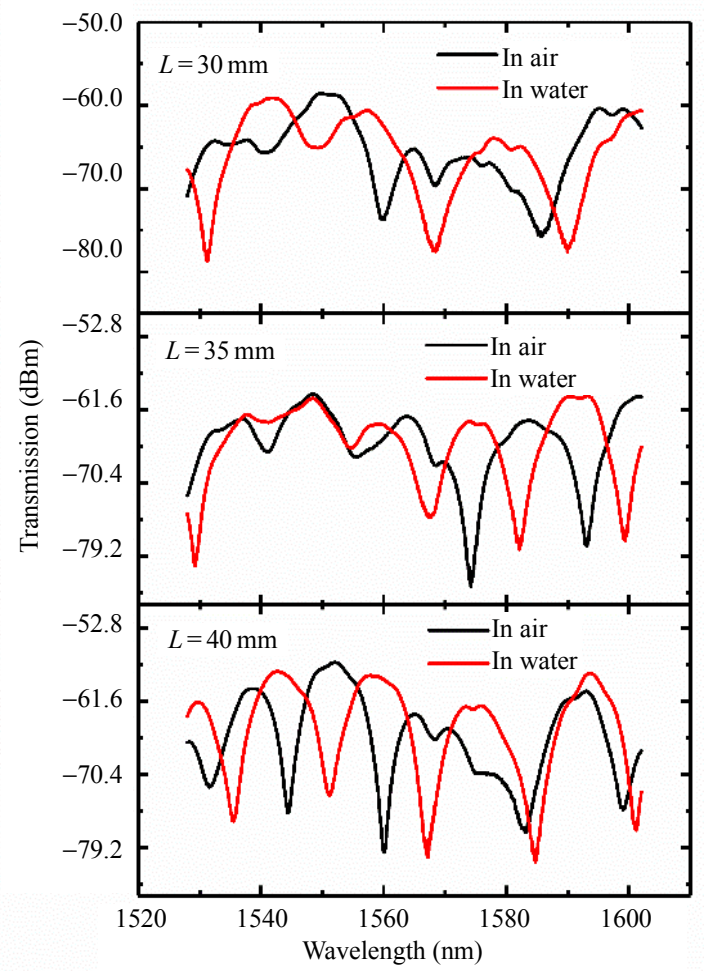

Fig. 3 Transmission spectra of a PCF interferometer with different sensing lengths in air and water.

The ability of the PCF interferometer to detect the change of RI in the liquid environment is studied. The refractive index solution is formed by mixing water with glycerol in a certain proportion, and it ranges from 1.333 to 1.381 in the experiments. Figures 4(a), 4(b), and 4(c) are the spectral change diagrams of three different lengths PCF interferometers in different refractive index liquids. With an increase in the refractive index, the wavelength of the interference spectrum appears red-shift. The relationship between the maximum wavelength shift amount and refractive index of three different lengths PCF interferometers are 
illustrated in Fig. 4(d) by linear fitting. The maximum refractive index sensitivities of the PCF interferometer with the interference length of $30 \mathrm{~mm}$, $35 \mathrm{~mm}$, and $40 \mathrm{~mm}$ are around $106.19 \mathrm{~nm} / \mathrm{RIU}$, $93.33 \mathrm{~nm} / \mathrm{RIU}$, and $73.643 \mathrm{~nm} / \mathrm{RIU}$, respectively, and

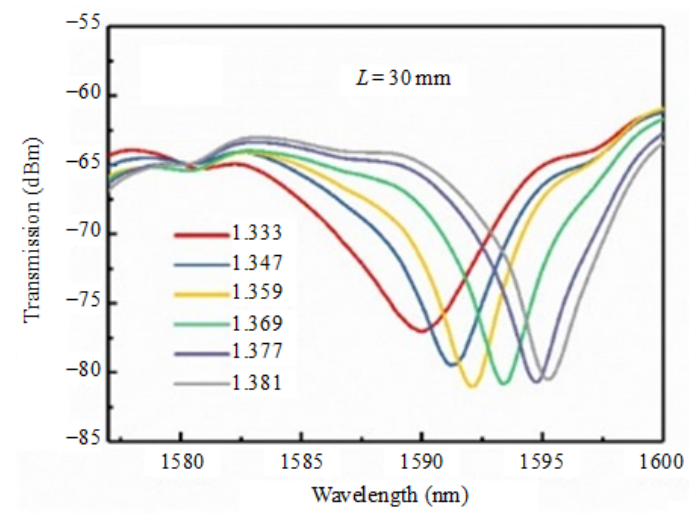

(a)

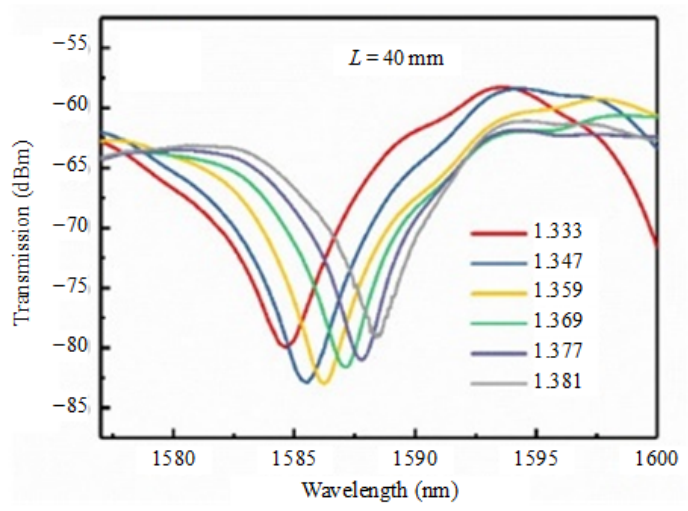

(c) the corresponding fitting degrees $\left(R^{2}\right)$ are 0.99234 , 0.99643 , and 0.98433 , respectively. The results show that the refractive index sensitivity of the PCF interferometer decreases with an increase in the interference length.

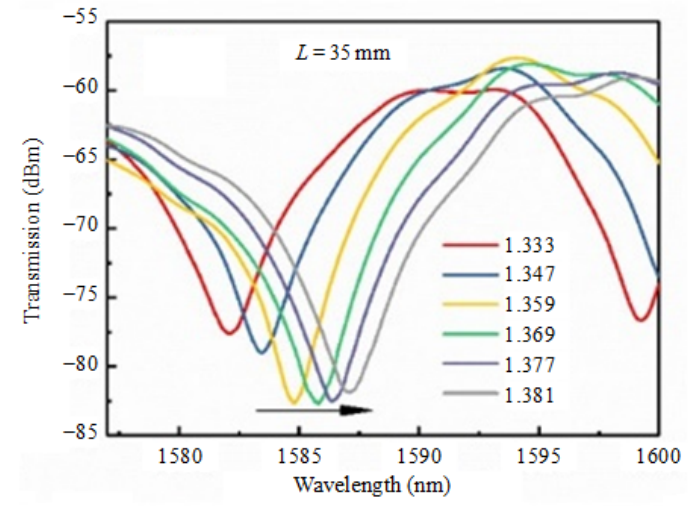

(b)

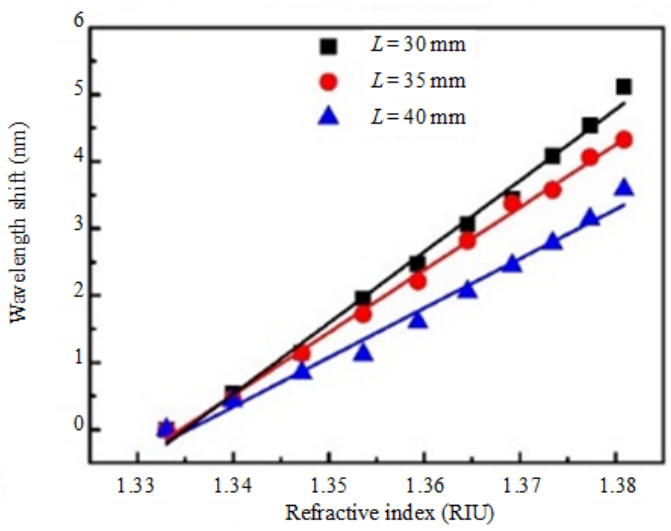

(d)

Fig. 4 Spectral change diagram of three different lengths PCF interferometers in different refractive index liquids (a) $L=30 \mathrm{~mm}$, (b) $L=35 \mathrm{~mm}$, (c) $L=40 \mathrm{~mm}$, and (d) relationship between the wavelength shift and surrounding RI of the PCF interferometer with different etching time.

In order to improve the refractive index sensitivity of the PCF interferometer, in this experiment, an HF etching method is used to corrode the PCF with a length of $35 \mathrm{~mm}$ because it has a better corresponding fitting degree, and the concentration of $\mathrm{HF}$ is $20 \%$. Figure 5 shows the different diameters of the PCF under the microscope, and the diameter is $125 \mu \mathrm{m}$ (no etching), $112 \mu \mathrm{m}$ (etching for $10 \mathrm{~min}$ ), and $91 \mu \mathrm{m}$ (etching for $80 \mathrm{~min}$ ), respectively. Figure 6 shows the transmission spectra of the MZI with different etching time in air. With an increase in the etching time, the wavelength of the interference spectrum appears blue-shift, and the transmission loss decreases gradually. Figures 7(a), (b), and 7(c) show the spectral change diagrams of the $35 \mathrm{~mm}$ MZI with different etching time when liquid RI varies. A red shift in the spectrum is observed when RI increases from 1.333 to 1.381 , and total wavelength shift amounts of the PCF interferometer before corrosion, and the etching 
time for $10 \mathrm{~min}$ and $80 \mathrm{~min}$ are $4.326 \mathrm{~nm}, 9.867 \mathrm{~nm}$, and $16.643 \mathrm{~nm}$, respectively. The RI sensitivities are obtained to be $93.33 \mathrm{~nm} / \mathrm{RIU}, 211.53 \mathrm{~nm} / \mathrm{RIU}$, and $359.37 \mathrm{~nm} / \mathrm{RIU}$ by linear fitting, and they have better corresponding fitting degrees $(0.99643,0.99553$, and 0.99661, respectively), as shown in Fig. 7(d). Considering the wavelength resolution $(10 \mathrm{pm})$ used by the OSA in this experiment, the resolution of RI index limit is $1.07 \times 10^{-4} \mathrm{RIU}, 4.73 \times 10^{-5} \mathrm{RIU}$, $2.78 \times 10^{-5}$ RIU, respectively, within the range from 1.333 to 1.381 .

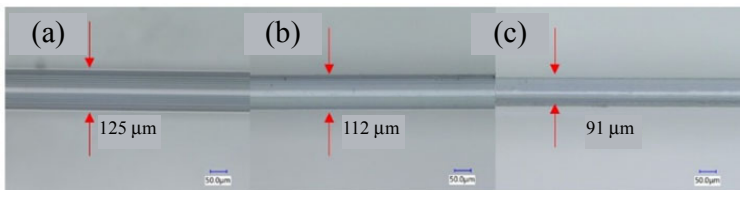

Fig. 5 Different diameter of PCF under the microscope.

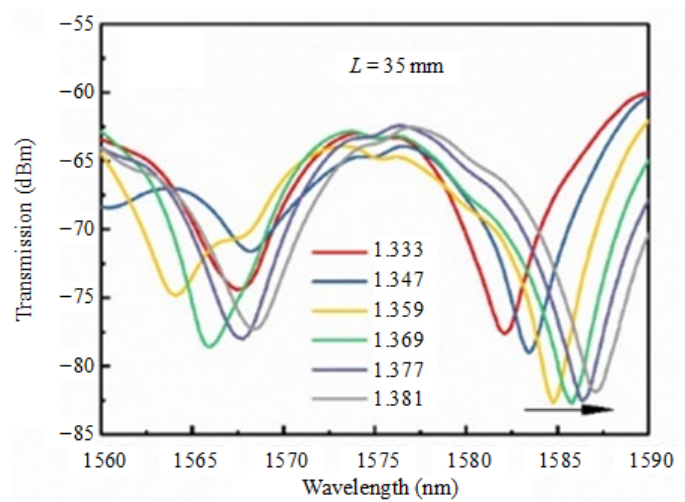

(a)

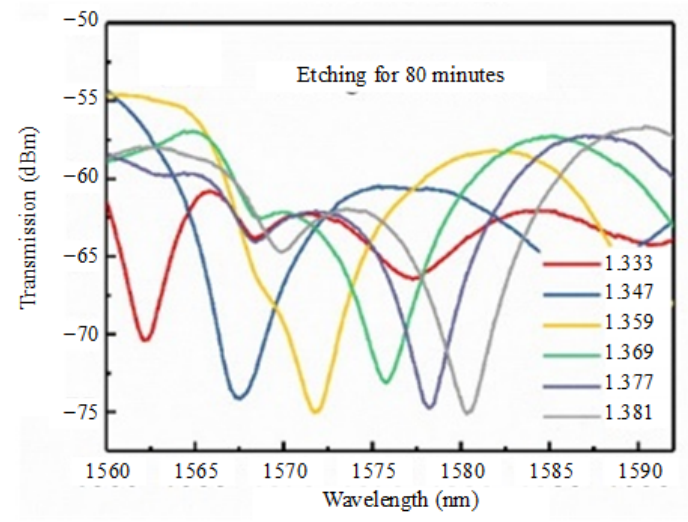

(c)

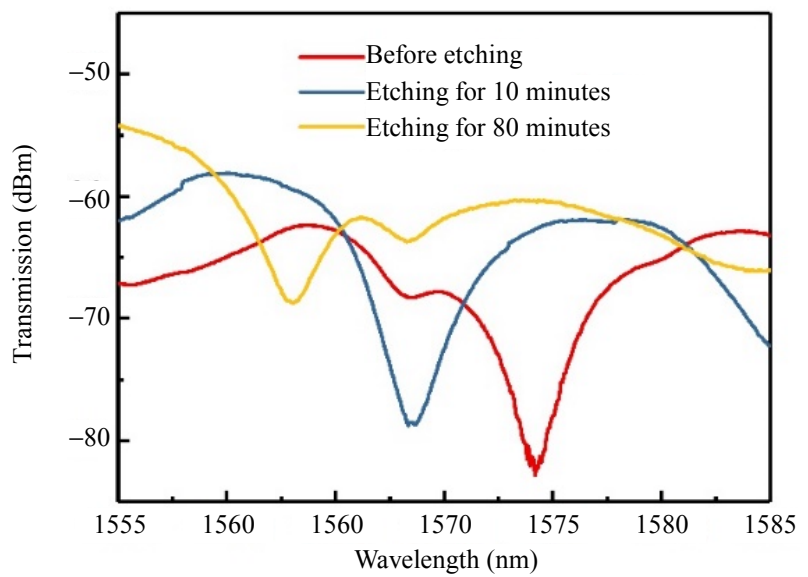

Fig. 6 Transmission spectra of PCF interferometer with different etching time in air.

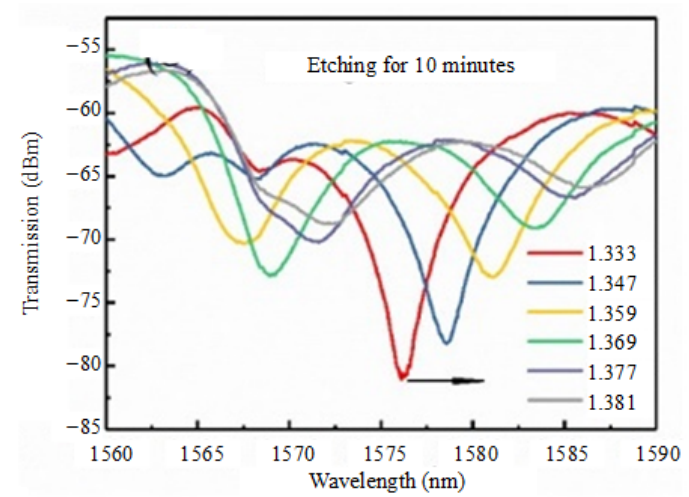

(b)

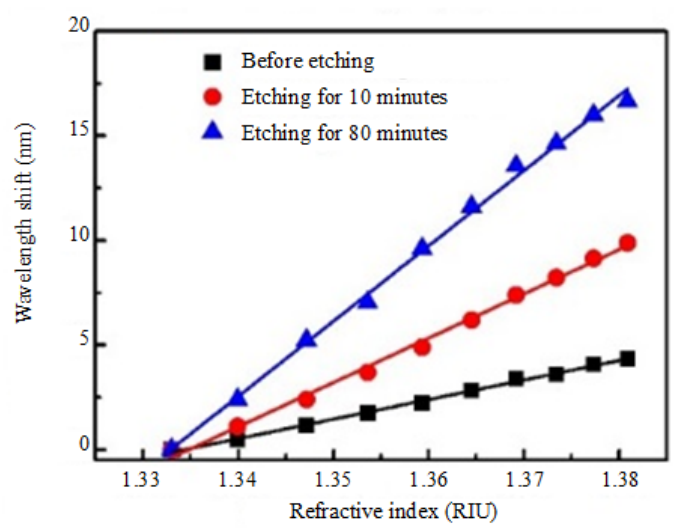

(d)

Fig. 7 Spectral change diagrams of (a) before corrosion, (b) etching for 10 minutes, (c) etching for 80 minutes, and (d) relationship between wavelength shift and surrounding RI of PCF interferometer with different etching time. 
It can be seen that the RI sensitivities of the PCF interferometer are improved evidently with an increase in the etching time. The reason is that the PCF diameter is decreased by the HF etching method. When the surrounding RI is a constant, a PCF diameter decrease will result in an increase in $\Delta n_{\text {eff }}$ as expressed in (5). That is to say, the interaction between the evanescent waves of the cladding modes and the ambient environment around the fiber will be enhanced, so the influence of the surrounding refractive index changing on the transmission characteristics of the cladding mode is more obvious.

Because the PCF has a micro-hole structure which has a small coefficient of thermal expansion, the main advantage of the PCF is that it is insensitive to the temperature. In order to prove that temperature has less influence on the refractive index measurement, a $35 \mathrm{~mm}$ PCF interferometer is heated from $20{ }^{\circ} \mathrm{C}$ to $500{ }^{\circ} \mathrm{C}$. The transmission spectra of this PCF interferometer are shown in Fig. 8(a). The relationship between the wavelength shift and temperature is shown in Fig. 8(b), and the temperature sensitivity is $9.21 \mathrm{pm} /{ }^{\circ} \mathrm{C}$. As the environmental temperature change is about $10{ }^{\circ} \mathrm{C}$, the corresponding wavelength shift of the PCF interferometer is less than $0.1 \mathrm{~nm}$. Our experimental temperature is $20^{\circ} \mathrm{C}$, and the range of temperature fluctuation is slight (within $2{ }^{\circ} \mathrm{C}$ fluctuation). This means that temperature has less influence on the refractive index measurement, which could be ignored compared with the RI sensitivity.

A comparison between the proposed MZI and related MZIs based on the splicing technology is listed in Table 1. Table 1 shows that the sensitivities of the proposed sensor have higher RI sensitivity than PCF with up-tapered joints $(252 \mathrm{~nm} / \mathrm{RIU})$ [14], PCF-MZI with two HTCRS (181.96nm/RIU) [16], and MMF (Multimode fiber)-SMF-MMF $(-37.93 \mathrm{~nm} / \mathrm{RIU})$ [25]. Because the diameter of the corroded PCF decreases, the interaction between the evanescent wave of cladding mode and the surrounding environment of the fiber is enhanced. However, the sensor we reported has lower sensitivity than the open-cavity MZI $(-1364.343 \mathrm{~nm} / \mathrm{RIU})$, but the open-cavity MZIs usually have small RI measurement range (only 1.333 - 1.3468) although they have ultrahigh RI sensitivity. Because the PCF is insensitive to the temperature, the temperature sensitivity of the proposed PCF interferometers $\left(9.21 \mathrm{pm} /{ }^{\circ} \mathrm{C}\right)$ is almost the same with that of other PCF structure interferometers, but they are lower than that of the SMF structure MZIs, such as small offset with the SMF $\left(49 \mathrm{pm} /{ }^{\circ} \mathrm{C}\right)[24]$ and open-cavity MZI [26].

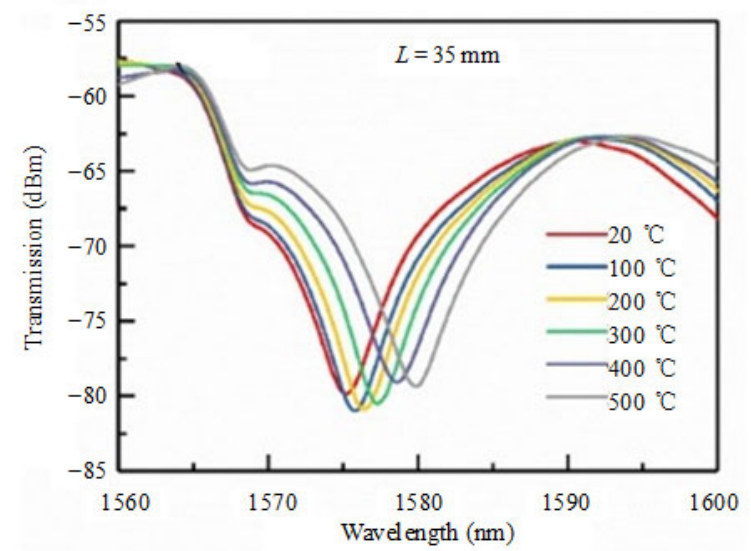

(a)

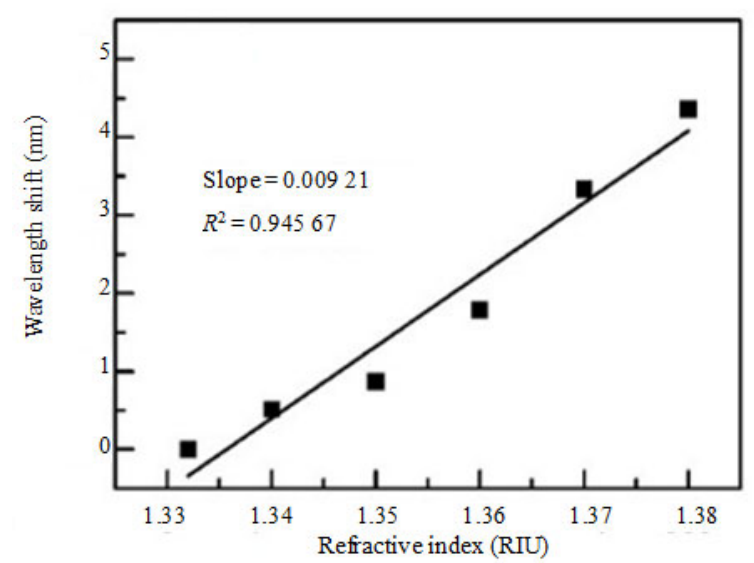

(b)

Fig. 8 Results of temperature response experiment: (a) transmission spectra of the PCF interferometer and (b) relationship between the wavelength shift and temperature. 
Table 1 Comparison between the available MZI sensors and the proposed MZI.

\begin{tabular}{cccccc}
\hline Sensor structure & Refractive sensitivity $(\mathrm{nm} / \mathrm{RIU})$ & Refractive range & Temperature sensitivity $\left(\mathrm{pm} /{ }^{\circ} \mathrm{C}\right)$ & Temperature range $\left({ }^{\circ} \mathrm{C}\right)$ & Reference \\
\hline PCF with up-tapered joints & 252 & $1.333-1.379$ & 2.96 & $20-170$ & {$[14]$} \\
PCF taper structure & 51.902 & $1.3411-1.3737$ & 0.9 & $20-50$ & {$[15]$} \\
PCF-MZI with two HTCRS & 181.96 & $1.3333-1.3574$ & - & - & {$[16]$} \\
MZI based on step-like tapers & -185.79 & $1.3333-1.3673$ & - & - & {$[23]$} \\
Small offset with SMF & -38.1 & $1.333-1.3739$ & 49 & 50 & {$[24]$} \\
MMF-SMF-MMF & -37.93 & $1.3105-1.3571$ & 52.2 & $25-85$ & {$[25]$} \\
open-cavity MZI & -1364.343 & $1.333-1.3468$ & 33 & $20-100$ & {$[26]$} \\
This work & 359.37 & $1.333-1.381$ & 9.21 & 2000 & \\
\hline
\end{tabular}

\section{Conclusions}

The paper proposes and studies a high sensitive refractive index sensor based on the Mach-Zehnder interferometer sensor, which splices a section of photonic crystal fiber (PCF) between two SMFs. The RI sensitivity of the MZI with the $35 \mathrm{~mm}$ PCF is enhanced obviously by HF etching with different time. In the RI range of 1.333 to 1.381 , the RI sensitivity is $93.33 \mathrm{~nm} / \mathrm{RIU}$ non-corrosion, which is up to $211.53 \mathrm{~nm} / \mathrm{RIU}$ and $359.37 \mathrm{~nm} / \mathrm{RIU}$ after $10 \mathrm{~min}$ and $80 \mathrm{~min} \mathrm{HF}$ corrosion, and the temperature sensitivity is $9.21 \mathrm{pm} /{ }^{\circ} \mathrm{C}$ with the temperature range from $20^{\circ} \mathrm{C}$ to $500^{\circ} \mathrm{C}$. In addition, the PCF-MZI has advantages of temperature insensitivity, simple fabrication, low cost, and high sensitivity, which is widely used in the biological and chemical environmental detection.

\section{Acknowledgement}

This research work is supported by the National Natural Science Foundation of China (NSFC) (Grant Nos. 51475482, 51875584, 51875585, and 51475481), the National Key R\&D Program of China (Grant Nos. 2017YFB1104800 and 2018YFB1107803), and the Fundamental Research Funds for the Central Universities of Central South University.

Open Access This article is distributed under the terms of the Creative Commons Attribution 4.0 International License (http://creativecommons.org/licenses/by/4.0/), which permits unrestricted use, distribution, and reproduction in any medium, provided you give appropriate credit to the original author(s) and the source, provide a link to the Creative Commons license, and indicate if changes were made.

\section{References}

[1] X. Y. Sun, X. R. Dong, Y. W. Hu, H. T. Li, D. K. Chu, J. Y. Zhou, et al., "A robust high refractive index sensitivity fiber Mach-Zehnder interferometer fabricated by femtosecond laser machining and chemical etching," Sensors \& Actuators A: Physical, 2015, 230: 111-116.

[2] J. H. Wo, G. H. Wang, Y. Cui, Q. Sun, R. B. Liang, P. Shum, et al., "Refractive index sensor using microfiber-based Mach-Zehnder interferometer," Optics Letters, 2012, 37(1): 67-69.

[3] Q. Wang, L. X. Kong, Y. L. Dang, F. Xia, Y. W. Zhang, Y. Zhao, et al., "High sensitivity refractive index sensor based on splicing points tapered SMF-PCF-SMF structure Mach-Zehnder mode interferometer," Sensors \& Actuators B: Chemical, 2016, 225: 213-220.

[4] J. N. Wang and J. L. Tang, "Photonic crystal fiber Mach-Zehnder interferometer for refractive index sensing," Sensors, 2012, 12(3): 2983-2995.

[5] B. Y. Li, L. Jiang, S. M. Wang, H. L. Tsai, and H. Xiao, "Femtosecond laser fabrication of long period fiber gratings and applications in refractive index sensing," Optics \& Laser Technology, 2011, 43(8): 1420-1423.

[6] F. Zou, Y. Q. Liu, C. L. Deng, Y. H. Dong, S. Zhu, and T. Y. Wang, "Refractive index sensitivity of nano-film coated long-period fiber gratings," Optics Express, 2015, 23(2): 1114-1124.

[7] W. Liang, Y. Huang, Y. Xu, R. K. Lee, and A. Yariv, "Highly sensitive fiber Bragg grating refractive index sensors," Applied Physics Letters, 2005, 86(15): 151122-1-151122-3. 
[8] T. Wei, Y. K. Han, Y. J. Li, H. L. Tsai, and H. Xiao, "Temperature-insensitive miniaturized fiber inline Fabry-Perot interferometer for highly sensitive refractive index measurement," Optics Express, 2008, 16(8): 5764-5769.

[9] X. Y. Sun, D. K. Chu, X. R. Dong, C. Zhou, H. T. Li, L. Zhi, et al., "Highly sensitive refractive index fiber inline Mach-Zehnder interferometer fabricated by femtosecond laser micromachining and chemical etching," Optics \& Laser Technology, 2016, 77: 11-15.

[10] P. Lu, L. Q. Men, K. Sooley, and Q. Chen, “Tapered fiber Mach-Zehnder interferometer for simultaneous measurement of refractive index and temperature," Applied Physics Letters, 2009, 94(13): 131110-1-131110-3.

[11] X. R. Dong, Z. Xie, Y. X. Song, K. Yin, D. K. Chu, and J. A. Duan, "High temperature-sensitivity sensor based on long period fiber grating inscribed with femtosecond laser transversal-scanning method," Chinese Optics Letters, 2017, 15(9): 090602-1-090602-5.

[12] X. R. Dong, Z. Xie, C. Zhou, K. Yin, Z. Luo, and J. A. Duan, "Temperature sensitivity enhancement of platinum-nanoparticle-coated long period fiber gratings fabricated by femtosecond laser," Applied Optics, 2017, 56(23): 6549-6553.

[13] T. A. Birks, J. C. Knight, and P. S. J. Russell, "Endlessly single-mode photonic crystal fiber," Optics Letters, 1997, 22(13): 961-963.

[14] Y. Zhao, X. G. Li, L. Cai, and Y. Yang, "Refractive index sensing based on photonic crystal fiber interferometer structure with up-tapered joints," Sensors \& Actuators B: Chemical, 2015, 221: 406-410.

[15] D. Wu, Y. Zhao, and J. Li, "PCF taper-based Mach-Zehnder interferometer for refractive index sensing in a PDMS detection cell," Sensors \& Actuators B: Chemical, 2015, 213: 1-4.

[16] Y. Zhao, F. Xia, H. F. Hu, and M. Q. Chen, "A novel photonic crystal fiber Mach-Zehnder interferometer for enhancing refractive index measurement sensitivity," Optics Communications, 2017, 402: 368-374.

[17] D. Pawar and S. N. Kale, "Birefringence manipulation in tapered polarization-maintaining photonic crystal fiber Mach-Zehnder interferometer for refractive index sensing," Sensors \& Actuators A: Physical, 2016, 252: 180-184.

[18] Y. C. Tan, Z. Q. Tou, V. Mamidala, K. K. Chow, and C. C. Chan, "Continuous refractive index sensing based on carbon-nanotube-deposited photonic crystal fibers," Sensors \& Actuators B: Chemical, 2014, 202: 1097-1102.

[19] L. Melo, G. Burton, P. Kubik, and P. Wild, "Refractive index sensor based on inline Mach-Zehnder interferometer coated with hafnium oxide by atomic layer deposition," Sensors and Actuators B: Chemical, 2016, 236: 537-545.

[20] T. Zhu, F. F. Xiao, L. C. Xu, M. Liu, M. Deng, and K. S. Chiang, "Pressure-assisted low-loss fusion splicing between photonic crystal fiber and single-mode fiber," Optics Express, 2012, 20(22): 24465-24471.

[21] D. K. Sharma and A. Sharma. "Mode field expansion in index-guiding microstructured optical fibers," SPIE, 2013, 8794: 87942A-1-87942A-6.

[22] L. C. Li, L. Xia, Z. H. Xie, and D. M. Liu, “All-fiber Mach-Zehnder interferometers for sensing applications," Optics Express, 2012, 20(10): 11109-11120.

[23] Y. Zhao, M. Q. Chen, F. Xia, and H. F. Hu, "Spectrum online-tunable Mach-Zehnder interferometer based on step-like tapers and its refractive index sensing characteristics," Optics Communications, 2017, 403: 143-149.

[24] L. Cai, Y. Zhao, and X. G. Li, "A fiber ring cavity laser sensor for refractive index and temperature measurement with core-offset modal interferometer as tunable filter," Sensors \& Actuators B: Chemical, 2017, 242: 673-678.

[25] R. Xiong, H. Y. Meng, Q. Q. Yao, B. Huang, Y. M. Liu, H. C. Xue, et al., "Simultaneous measurement of refractive index and temperature based on modal interference," IEEE Sensors Journal, 2014, 14(8): 2524-2528.

[26] L. Q. Wang, L. Yang, C. Zhang, C. Y. Miao, J. F. Zhao, and W. Xu, "High sensitivity and low loss open-cavity Mach-Zehnder interferometer based on multimode interference coupling for refractive index measurement," Optics and Laser Technology, 2019, 109: 193-198. 